\title{
In Situ Bioremediation of Contaminated Soil and Ground Water; Lab Optimization Leads to Functional Combinations at Field Sites
}

\author{
Martin Romantschuk \\ Faculty of Biological and Environmental Sciences, Environmental Biotechnology \\ University of Helsinki, Finland \\ martin.romantschuk@helsinki.fi
}

\section{Background}

Organic contaminants in the soil is a widespread problem that not only may cause damage to local biota, but also poses an ecological and health threat if the contaminants spread to groundwater aquifers and surface waters. Therefore, sites known to be contaminated should always be assessed preferable by performing both an ecological risk assessment and a health risk assessment. Based on the results of such assessments remediation decisions regarding remediation activities are made. One aim is to optimize the outcome and cost efficiency. For more than 20 years we have developed and tested approaches for enhancement of bioremediation. Through collaboration between research facilities, contractors, and site owners, more than 30 actual sites presenting typical problems have been targets for testing and optimization, first by laboratory modeling, and then by applying lab experiences in pilot scale and application full scale.

\section{Aim}

Through national and international collaboration we aim to recognize as many viable methods for in situ remediation as possible, and to test these methods in lab as well as in pilot and application scale together with expert scientists and contractors. By using established and novel methods in new innovative combinations we hope to be able to find functional in situ solutions for almost any contamination situation. Our aim is also to convince public authorities along with contractors to accept and welcome novel methods. In situ solutions should become the norm rather that a curiosity.

\section{Conclusion}

Samples from various types of contaminated sites and conditions have been used in controlled laboratory conditions to build micro- and mesocosm- setups in which biological, physical, and chemical treatments are either combined or used in sequence. Vital for success is a thorough knowledge of the site, and therefore all site characterization efforts preceding remediation activities are crucial. In most cases the goal is to achieve optimal biostimulation and contaminant degradation. This is achieved by optimizing conditions for microbial activity, and by improving bioavailability of the target compounds. However, when needed also chemical and physical means are harnessed. Along these lines we have developed novel forms of underground flotation for removal of volatile compounds from soil and ground water. Further, we use electrokinetic pumping in combinations with biostimulation, bioaugmentation, nanoremediation etc. for maximal spreading of the intended effect. At actual sites we have proven the functionality of a number of combinations, and additional ones are tested presently. Several SMEs are involved in our activities, and contacts with public authorities are dynamic. 